\title{
Traumatismo craneoencefálico en la ciudad de Buenos Aires: estudio epidemiológico prospectivo de base poblacional
}

\author{
P.S. Marchio; I.J. Previgliano*; C.E. Goldini y F. Murillo-Cabezas**
}

Área de Emergencia y Terapia Intensiva. *Terapia Intensiva. Hospital General de Agudos J.A. Fernández. Cerviño. Buenos Aires. Argentina **Servicio de Cuidados Críticos y Urgencias. Hospital Universitario Virgen del Rocío. Sevilla. España.

\section{Resumen}

Objetivo. El propósito de este trabajo es mostrar la epidemiología del traumatismo craneoencefálico (TCE) en una población de la ciudad de Buenos Aires, cuyas características socioeconómicas representan a todos los sectores de la sociedad.

Material y métodos. Estudio prospectivo, de base poblacional, autorizado por el Comité de Ética y de Docencia e Investigación como parte de un programa integral de neurotrauma.

Resultados. Tasa de incidencia de 322 TCE por 100.000 habitantes. Un $93 \%$ TCE leves; $4 \%$ TCE moderados y 3\% TCE graves. EI promedio de edad de las mujeres fue mayor que la de los hombres (49 vs 38 años, $p<0,01)$. La población menor de 40 años con respecto a los mayores de 40 años presentó: un riesgo relativo (RR) 1,97 veces mayor de sufrir cualquier tipo de TCE (IC 95\% 1,77 - 2,19, p < 0,01). Los menores de 40 años también, mostraron un RR de 2,53 (IC 95\% 2,03 - 3,17, $\mathbf{p}<\mathbf{0 , 0 1 )}$ para sufrir un TCE por vehículos a motor. Los conductores de auto presentaron un RR de 16,76 (IC $95 \% 5,35-52,50, p<0,01)$ de sufrir un accidente siendo hombre, independientemente de la edad. La agresión fue también mayor en los menores de 40 años, con un RR de 2,11 (IC 95\% 1,46 - 3,03, p < 0,01) que aumenta cuando se diferencia por sexos. Para las caídas de la propia altura, en cambio, el RR fue de 4,35 (IC 95\% $\mathbf{2 , 6 1}-4,16, p<0,01)$ para los mayores de 40 años y de 3,15 (IC 95\% 2,57 - 3,87, p < 0,01) para las mujeres independientemente de la edad. Asimismo, el RR para sufrir un atropello fue más alto para los mayores de $\mathbf{4 0}$ años $(1,84$ IC $95 \% 1,41-2,41 p<0,01)$. No se encontraron otras diferencias estadísticas ni aumento del $R R$ en los demás mecanismos de trauma.

Conclusiones. La incidencia de TCE es elevada, con una proporción de TCC moderados y graves y tasa de mortalidad inferior a otras series. Los accidentes de tráfico continúan como primer agente causal, afectando,

Recibido: 05-10-04. Aceptado: 11-02-05. sobre todo, a la población joven masculina, mientras las caídas de altura y atropellos inciden en la población mayor de 40 años y sexo femenino.

PALABRAS CLAVE: Traumatismo craneoencefálico. Epidemiología. Incidencia. Factores de riesgo.

Head injury in Buenos Aires city: a prospective, population based, epidemiologic study

Summary

Objective. The aim of the study is to present head injury (HI) epidemiology in a population of Buenos Aires that represents almost all socioeconomic sectors.

Material and method. Prospective, population based study, approved by the Ethic, Education and Research Committees as part of a neurotrauma program.

Results. HI incidence was $322 / 100000$ inhabitants, of them mild HI accounted for $93 \%$, moderate HI $4 \%$ and severe $\mathrm{HI} 3 \%$. Average age was greater in women than in men ( 49 vs. 38 yo, p 0,01). Taking into account population under and over 40 years we found several differences: the relative risk (RR) for any kind of $\mathrm{HI}$ was 1,97 (CI 95\% 1,77 - 2,19, p < 0,01, and the RR for motor vehicle (MV) related HI was 2,53 (CI 95\% 2,03 $-3,17, p<0,01)$. Male drivers had a higher RR $(16,76 \mathrm{CI}$ $95 \% 5,35-52,50, p<0,01)$ regardless age. Assaults were also significantly higher in people under 40 yo (RR 2,11 IC 95\% 1,46 - 3,03, p < 0,01) increased in young males.

Self altitude falls were more frequent in population over 40 yo (RR 4,35 CI 95\% 2,61 - 4,16, p < 0,01) and in women at any age (RR 3,15 CI 95\% 2,57 - 3,87, p $<0,01)$. Pedestrian accidents were also more common in population over 40 yo (RR 1,84 CI 95\% 1,41 -2,41 p $<0,01$ ).

We did not found any other differences concerning

Abreviaturas. GCS: Glasgow coma scale. HI: head injury. RR: riesgo relativo. SAME sistemas de emergencia. TCE: traumatismo craneoencefálico. 
the remaining trauma mechanisms.

Conclusions. HI incidence in Buenos Aires is similar to other printed series, but moderate and severe $\mathrm{HI}$ and mortality rates are below that ones. Motor vehicle accidents are the leading HI cause, especially in the young male population, while self altitude fall and pedestrian accidents are prevalent in women and people over 40 yo.

KEY WORDS: Traumatic brain injury. Head injury. Epidemiology. Incidence. Risk factors.

\section{Introducción}

El traumatismo craneoencefálico (TCE), también llamado la "epidemia silenciosa", es la principal causa de muerte e incapacidad en individuos menores de 40 años. Su enorme repercusión socio sanitaria ha propiciado numerosos estudios epidemiológicos en distintos países ${ }^{1,3,6}$, entre ellos España ${ }^{4}$, sobre todo en la década de los 70 y 80 . Asimismo, los costes económicos son muy elevados. Un estudio reciente referido al coste económico de los accidentes de tráfico en España ha estimado su valor en el año 1997 en 6.280.36 millones de euros, lo que representa el 1,35\% del producto interior bruto ${ }^{10}$. En Argentina, en cambio, no se ha realizado ningún estudio de esta naturaleza. La carencia de datos en nuestro país, unido a los cambios que se están produciendo en todo el mundo en el perfil epidemiológico del TCE nos ha movido al presente estudio.

El propósito de este trabajo es conocer la epidemiología del TCE en Argentina, a través de los datos obtenidos en una población definida, cuyas características demográficas, sociales y económicas representan a todos los sectores de la sociedad.

\section{Material y método}

\section{Diseño del estudio}

Epidemiológico, observacional, prospectivo, de base poblacional. El estudio fue aprobado por los Comités de Ética y Docencia e Investigación como parte del programa del Grupo de Trabajo en Neurotrauma.

\section{Ámbito}

Población de referencia del Hospital General de Agudos, J. A. Fernández, del Gobierno de la Ciudad de Buenos Aires, que comprende un total de 478.700 habitantes, de los cuales 31.200 habitan en barrios marginales y 2.800 habitan en "casas ocupadas" (casas tomadas).

Recogida de Dalos
Diariamente, desde el 1 de Octubre de 2000 al 30 de Septiembre de 2001, se recogieron los siguientes datos: sexo, edad, fecha, hora y mecanismo del accidente, puntuación inicial en la Escala de Coma de Glasgow (GCS), factores de riesgo, de acuerdo con las Guías para el Manejo del Traumatismo de Cráneo Leve en Adultos de la Sociedad Italiana de Neurocirugía ${ }^{18}$, presencia de aliento etílico o intoxicación alcohólica, lesiones en la tomografía computarizada craneal (TAC) clasificadas de acuerdo al Traumatic Coma Data Bank ${ }^{6,12}$, tiempo de observación en el área de emergencias, destino inicial (alta, ingreso en planta, ingreso en la unidad de cuidados intensivos (UCI), derivación a otro centro asistencial, fuga o muerte a la admisión) y destino final (alta domiciliaria, derivación a otro centro, fuga o muerte).

De acuerdo a la puntuación en la GCS, se clasificó a los TCE en leves (14-15), moderados (9-13) y graves (38). Los datos se relacionaron con la población del área asistencial y se expresan por 100.000 habitantes, por sexo y edad. Se estableció la relación hombres/mujeres para el total de la población y para cada grupo de edad. Se buscaron diferencias entre los mecanismos y gravedad de trauma de acuerdo a sexo y edad, tomando como punto de corte los 40 años. Este punto fue elegido teniendo en cuenta los hallazgos publicados en las Guías de la Brain Trauma Foundation $^{11}$

\section{Análisis estadístico}

Los datos estudiados fueron registrados y analizados en una hoja de cálculo de Excel $^{\circledR}$. Se utilizó el test de Chi cuadrado para el cálculo de las diferencias de variables discretas, tasa de probabilidad (OR) y riesgo relativo (RR). Se utilizó el test de la T de Student con dos colas para diferencia de medias con varianzas iguales o desiguales. Se consideró significativo un valor de $\mathrm{p}<0.05$.

\section{Resultados}

Durante el período de estudio establecido, se registraron datos de 1.540 pacientes con TCE, que correspondieron al 2,5\% de un total de 58.104 consultas realizadas en el mismo período en el Departamento de Urgencias. De estos pacientes $93 \%$ se clasificaron como TCE leve; $4 \%$ como TCE moderado y $3 \%$ como TCE grave. En la tabla 1 se muestra la incidencia por 100.000 habitantes. La incidencia total fue de 322 por 100.000 habitantes, correspondiendo 300 a TCE leve, 13 a TCE moderado y 9 a TCE grave por 100.000 habitantes respectivamente. Nótese que la distribución (Figura 1) no es gaussiana, con picos de incidencia entre los 20 y 24 años; 40 y 44 años; y más de 75 años. En la tabla 2 se observa la incidencia total por sexo y edad. En la tabla 3 se indica la relación hombre/mujer para todo el 


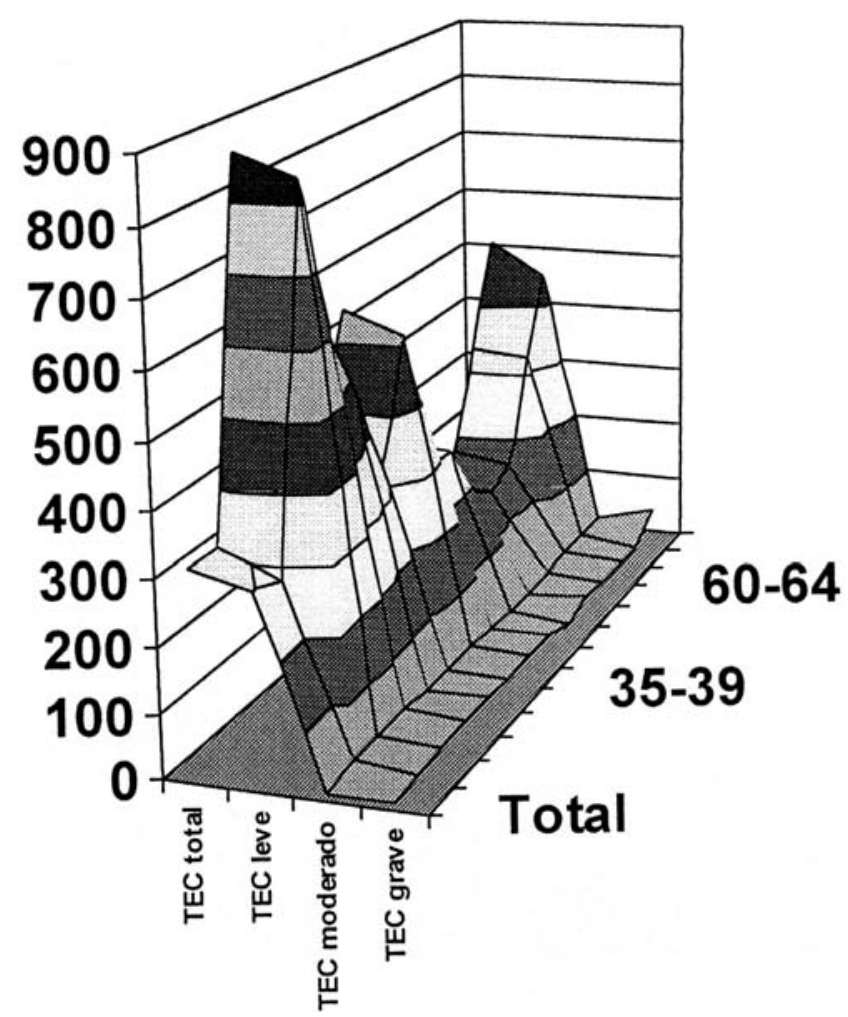

\begin{tabular}{|l|}
\hline$\quad 800-900$ \\
$\square 700-800$ \\
$\square 600-700$ \\
$\square 500-600$ \\
$\square 400-500$ \\
$\square 300-400$ \\
$\square 200-300$ \\
$\square 100-200$ \\
$\square 0-100$ \\
\hline
\end{tabular}

Figura 1. Incidencia de TCE por cada 100.000 habitantes. Nótese la distribución no gaussiana, con picos entre los 20 , 40 y 75 años. Estas variaciones son secundarias al TCE leve, ya que la incidencia de TCE moderado y grave se mantiene relativamente constante.

Tabla 1

Incidencia de TCE por cada 100.000 habitantes de acuerdo a edad

\begin{tabular}{|c|c|c|c|c|}
\hline & TCE total & TCE leve & TCE moderado & TCE grave \\
\hline Total & 322 & 301 & 13 & 9 \\
\hline $15-19$ & 321 & 283 & 24 & 14 \\
\hline $20-24$ & 868 & 836 & 20 & 12 \\
\hline $25-29$ & 630 & 586 & 26 & 18 \\
\hline $30-34$ & 499 & 464 & 16 & 19 \\
\hline $35-39$ & 356 & 331 & 13 & 13 \\
\hline $40-44$ & 325 & 291 & 20 & 13 \\
\hline $45-49$ & 301 & 273 & 14 & 14 \\
\hline $50-54$ & 277 & 270 & 7 & 0 \\
\hline $55-59$ & 186 & 169 & 7 & 10 \\
\hline $60-64$ & 152 & 141 & 10 & 0 \\
\hline $65-69$ & 179 & 162 & 12 & 4 \\
\hline $70-74$ & 336 & 323 & 14 & 0 \\
\hline $75-$ & 500 & 449 & 13 & 38 \\
\hline
\end{tabular}


Tabla 2

Incidencia de TCE según sexo, edad y gravedad por cada 100.000 habitantes

\begin{tabular}{|c|c|c|c|c|c|c|c|c|}
\hline & $\begin{array}{c}\text { TCE } \\
\text { Mujeres }\end{array}$ & $\begin{array}{l}\text { TCE } \\
\text { Hombres }\end{array}$ & $\begin{array}{l}\text { TCE } \\
\text { leve } \\
\text { Mujeres }\end{array}$ & $\begin{array}{l}\text { TCE } \\
\text { leve } \\
\text { Hombres }\end{array}$ & \begin{tabular}{l}
\multicolumn{1}{c}{ TCE } \\
moderado \\
Mujeres
\end{tabular} & $\begin{array}{l}\text { TCE } \\
\text { moderado } \\
\text { Hombres }\end{array}$ & $\begin{array}{l}\text { TCE } \\
\text { grave } \\
\text { Mujeres }\end{array}$ & $\begin{array}{c}\text { TCE } \\
\text { grave } \\
\text { Hombres }\end{array}$ \\
\hline Total & 124 & 558 & 116 & 520 & 3 & 23 & 4 & 15 \\
\hline $\begin{array}{l}15- \\
19\end{array}$ & 28 & 619 & 14 & 555 & 0 & 49 & 14 & 14 \\
\hline $\begin{array}{l}20- \\
24\end{array}$ & 153 & 1622 & 142 & 1568 & 11 & 30 & 0 & 24 \\
\hline $\begin{array}{l}25- \\
29\end{array}$ & 212 & 1065 & 172 & 1018 & 6 & 48 & 11 & 0 \\
\hline $\begin{array}{l}30- \\
34\end{array}$ & 95 & 965 & 95 & 890 & 0 & 34 & 0 & 41 \\
\hline $\begin{array}{l}35- \\
39\end{array}$ & 59 & 707 & 53 & 658 & 0 & 28 & 6 & 21 \\
\hline $\begin{array}{l}40- \\
44\end{array}$ & 37 & 676 & 30 & 610 & 0 & 45 & 6 & 22 \\
\hline $\begin{array}{l}45- \\
49\end{array}$ & 104 & 545 & 104 & 481 & 0 & 32 & 0 & 32 \\
\hline $\begin{array}{l}50- \\
54\end{array}$ & 150 & 432 & 150 & 417 & 0 & 15 & 0 & 0 \\
\hline $\begin{array}{l}55- \\
59\end{array}$ & 144 & 240 & 144 & 203 & 0 & 15 & 0 & 23 \\
\hline $\begin{array}{l}60- \\
64 \\
\end{array}$ & 60 & 276 & 48 & 268 & 12 & 8 & 0 & 0 \\
\hline $\begin{array}{l}65- \\
69\end{array}$ & 133 & 245 & 133 & 204 & 0 & 31 & 0 & 10 \\
\hline $\begin{array}{l}70- \\
74\end{array}$ & 179 & 592 & 171 & 568 & 7 & 24 & 7 & 0 \\
\hline 75- & 454 & 593 & 400 & 545 & 14 & 10 & 14 & 38 \\
\hline
\end{tabular}

grupo y para cada franja de edad. El promedio es de 4,5:1, con grandes variaciones en los menores de 30 años y 1,3:1 en los mayores de 70 años. La media de edad de las mujeres fue significativamente mayor que la de los varones (49 vs. $37, \mathrm{p}<0.01$ Student para varianzas desiguales).

Sólo en 5 casos el TCE tuvo como origen una lesión penetrante, por arma de fuego, y 261 casos presentaron lesiones asociadas. Con respecto al horario del trauma el $30 \%$ ocurrió de 8 a 16 horas; el 39\% de 16 a 0 horas; y el $31 \%$ de 0 a 8 horas. En 59 TCE se identificaron factores de riesgo de acuerdo a las Guías de la Sociedad Italiana de Neurocirugía. En 121 pacientes (8\%) se relacionó el TCE con el alcohol, ya fuera por aliento etílico u otros signos de intoxicación alcohólica aguda. En la tabla 4 se muestra la distribución por mecanismo de trauma y por sexo en núme- ros absolutos y porcentaje. Al final de la tabla se agrupan como accidentes de tráfico todos los implicados en ellos ya fueran conductores de automóviles y motos, acompañantes o peatones. Se advierte una clara diferencia en el mecanismo del trauma entre los menores y mayores de 40 años, que se observa en la tabla 5, por lo que se realizó el análisis para cada uno de esos grupos.

El análisis estadístico reveló que la población menor de 40 años respecto a la mayor de 40 años tiene: un RR 1,97 veces mayor de sufrir cualquier tipo de TCE (IC 95\%) 1,77 - 2,19, OR 1,97, p < 0,01); un RR de 2 (IC 95\% 1,79-2,23, OR 2,2, p < 0,01) para sufrir un TCE leve y un RR de 1,84 para un TCE moderado (IC 95\% 1,06 - 3,20, OR 1.87, p < $0,02)$. En el TCE grave, aunque se aprecia un aumento del RR de 1,45 para los menores de 40 años, éste no alcanza 
Tabla 3

Relación de incidencia de TCE entre hombres y mujeres por cada $\mathbf{1 0 0 . 0 0 0}$ habitantes, para el grupo en total y para cada franja de edad

\begin{tabular}{|c|c|}
\hline & Relación hombres/mujeres \\
\hline Total & 4,51 \\
\hline $15-19$ & 22,28 \\
\hline $20-24$ & 10,58 \\
\hline $25-29$ & 5,03 \\
\hline $30-34$ & 10,17 \\
\hline $35-39$ & 11,92 \\
\hline $40-44$ & 18,50 \\
\hline $45-49$ & 5,23 \\
\hline $50-54$ & 2,87 \\
\hline $55-59$ & 1,67 \\
\hline $60-64$ & 4,60 \\
\hline $65-69$ & 1,85 \\
\hline $70-74$ & 3,31 \\
\hline $75-$ & 1,31 \\
\hline
\end{tabular}

significación estadística, debido, probablemente, a cortedad de la muestra, como lo evidencia el aumento del IC $95 \%(0,80-2,63$, OR $1.45, \mathrm{p} 0,21)$

En cuanto al mecanismo de trauma, los menores de 40 años con relación a los que superan dicho tramo de edad tienen un RR 2,53 veces mayor (IC 95\% 2,03 - 3,17, p < $0,01)$ de tener un accidente de tráfico. Disminuye el RR, cuando se toma en cuenta la condición de conductor de automóvil y acompañante (RR 1,47 IC 95\% 1,14 - 1,91, $\mathrm{p}<0,01)$, aumentando cuando se analiza, aisladamente, conductor de moto (RR 14,23 IC 95\% 6,69 - 30,28, p < $0,01)$. Si se examina conductor de automóvil, exclusivamente, se observa un RR de 16,76 (IC 95\% 5,35 - 52,50, p $<0,01)$ veces mayor de sufrir un accidente siendo hombre, independientemente de la edad. La agresión interpersonal es también mayor en los menores de 40 años, con un RR de 2,11 (IC 95\% 1,46 - 3,03 p < 0,01) que se incrementa en el sexo masculino. Para las caídas de la propia altura, en cambio, el RR es de 4,35 (IC 95\% 2,61 - 4,16, p < 0.01) para los mayores de 40 años y de 3,15 (IC 95\% 2,57 - 3,87, $\mathrm{p}<0.01)$ para las mujeres independientemente de la edad. El RR para atropello de peatones es también mayor para los mayores de 40 años (1,84 IC 95\% 1,41 -2,41 p < 0.01). No se encontraron otras diferencias estadísticas ni aumento del RR en los demás mecanismos de trauma.

Se realizaron 219 TAC, cuyos resultados se muestran en la tabla 6. La incidencia de hematomas intracraneales fue del $1,6 \%$ y su localización se resume en la tabla 7 .

Tabla 4

Mecanismo del trauma del grupo general y de acuerdo a sexo, en números absolutos y porcentajes. Es importante notar la baja incidencia de mujeres conductoras accidentadas respecto de los varones, así como la mayor incidencia de caída (caída de propia altura) en las mujeres

\begin{tabular}{|l|c|c|c|c|c|c|c|}
\hline Mecanismo & Total & $\mathbf{\%}$ & Hombres & $\mathbf{\%}$ & Mujeres & $\mathbf{\%}$ & Valor de p \\
\hline Tráfico & $\mathbf{5 8 0}$ & $\mathbf{3 7 . 6}$ & $\mathbf{4 1 9}$ & $\mathbf{3 4 . 4}$ & $\mathbf{1 6 1}$ & $\mathbf{5 0}$ & $<\mathbf{0 . 0 1}$ \\
\hline Conductor & 114 & 7.4 & 111 & 10.6 & 3 & 0.6 & $<0.01$ \\
\hline Acompañante & 104 & 6,7 & 45 & 4.3 & 59 & 11.8 & $<0.01$ \\
\hline Moto & 124 & 8 & 111 & 10.6 & 13 & 2.6 & $<0.01$ \\
\hline Peatón & 196 & 12.7 & 120 & 11.5 & 76 & 15.2 & $<0.01$ \\
\hline Ciclista & 42 & 2.7 & 32 & 3.1 & 10 & 2 & $<0.01$ \\
\hline Caída & $\mathbf{2 9 9}$ & $\mathbf{1 9 . 4}$ & $\mathbf{1 1 9}$ & $\mathbf{1 1 . 4}$ & $\mathbf{1 8 0}$ & $\mathbf{3 6}$ & $<\mathbf{0 . 0 1}$ \\
\hline Precipitación & $\mathbf{1 1 3}$ & $\mathbf{7 . 4}$ & $\mathbf{7 7}$ & $\mathbf{1 3 . 1}$ & $\mathbf{3 6}$ & $\mathbf{7 . 2}$ & $<\mathbf{0 . 0 1}$ \\
\hline Convulsiones & $\mathbf{3}$ & $\mathbf{0 . 2}$ & $\mathbf{3}$ & $\mathbf{0 . 3}$ & $\mathbf{0}$ & $\mathbf{0}$ & $\mathbf{0 . 8 9}$ \\
\hline Herida & $\mathbf{8 6}$ & $\mathbf{5 . 6}$ & $\mathbf{6 6}$ & $\mathbf{6 . 3}$ & $\mathbf{2 0}$ & $\mathbf{4}$ & $\mathbf{0 . 4 2}$ \\
\hline Golpe & $\mathbf{2 1 9}$ & $\mathbf{1 4 . 2}$ & $\mathbf{1 7 0}$ & $\mathbf{1 6 . 3}$ & $\mathbf{4 9}$ & $\mathbf{9 . 8}$ & $<\mathbf{0 . 0 1}$ \\
\hline Agresión & $\mathbf{1 6 8}$ & $\mathbf{1 1}$ & $\mathbf{1 4 4}$ & $\mathbf{1 3 . 8}$ & $\mathbf{2 4}$ & $\mathbf{4 . 8}$ & $<\mathbf{0 . 0 1}$ \\
\hline Desconocido & $\mathbf{7 4}$ & $\mathbf{4 . 8}$ & $\mathbf{4 4}$ & $\mathbf{4 . 2}$ & $\mathbf{1 3 0}$ & $\mathbf{6}$ & $<\mathbf{0 . 0 1}$ \\
\hline
\end{tabular}


Tabla 5

Mecanismos del trauma según grupos de edad

\begin{tabular}{|l|c|c|c|c|c|}
\hline Mecanismo & Menor 40 años & $\mathbf{\%}$ & Mayor 40 años & $\mathbf{\%}$ & Valor de p \\
\hline Tráfico & $\mathbf{3 7 0}$ & $\mathbf{4 2 . 3}$ & $\mathbf{2 1 0}$ & $\mathbf{3 9 . 1}$ & $\mathbf{0 . 2 5}$ \\
\hline Acompañante & 79 & 9 & 25 & 4.6 & $<0.0$ \\
\hline Conductor & 62 & 7 & 52 & 9.6 & 0.10 \\
\hline Moto & 117 & 13.3 & 7 & 1.3 & $<0.01$ \\
\hline Peatón & 79 & 9 & 117 & 21 & $<0.01$ \\
\hline Ciclista & 33 & 3.7 & 9 & 1.6 & $<0.01$ \\
\hline Caída & $\mathbf{8 2}$ & $\mathbf{9 . 3}$ & $\mathbf{2 1 7}$ & $\mathbf{4 0 . 4}$ & $<\mathbf{0 . 0 1}$ \\
\hline Precipitación & $\mathbf{5 1}$ & $\mathbf{5 . 8}$ & $\mathbf{6 2}$ & $\mathbf{1 1 . 5}$ & $\mathbf{0 . 0 1}$ \\
\hline Convulsiones & $\mathbf{3}$ & $\mathbf{0}$ & $\mathbf{0}$ & $\mathbf{0}$ & $\mathbf{0 . 5 9}$ \\
\hline Herida & $\mathbf{6 1}$ & $\mathbf{6 . 9}$ & $\mathbf{2 5}$ & $\mathbf{4 . 6}$ & $\mathbf{0 . 0 9}$ \\
\hline Golpe & $\mathbf{1 4 3}$ & $\mathbf{1 3 . 3}$ & $\mathbf{1 7 6}$ & $\mathbf{1 3 . 2}$ & $\mathbf{0 . 2 2}$ \\
\hline Agresión & $\mathbf{1 1 9}$ & $\mathbf{2 . 9}$ & $\mathbf{4 9}$ & $\mathbf{8 . 5}$ & $<\mathbf{0 . 0 1}$ \\
\hline Desconocido & $\mathbf{2 6}$ & $\mathbf{4 8}$ & $\mathbf{8 . 9}$ & $<\mathbf{0 . 0 1}$ \\
\hline
\end{tabular}

Tabla 6

Clasificación de los hallazgos tomográficos según el Traumatic Coma Data Bank, expresados en números absolutos y porcentaje del total. Los totales se expresan como porcentaje del total de la categoría

\begin{tabular}{|l|c|c|c|c|c|c|}
\hline TCDB & TCE leve & $\%$ & TCE Moderado & $\%$ & TCE grave & $\%$ \\
\hline 1 (Normal) & 108 & 81.8 & 17 & 31 & 4 & 11.4 \\
\hline 2 (Difusa) & 20 & 15.1 & 13 & 24 & 6 & 17.1 \\
\hline 3 (Swelling bilateral) & 1 & 0 & 5 & 9.2 & 5 & 14.2 \\
\hline 4 (Swelling unilateral) & 0 & 0 & 0 & 0 & 0 & 0 \\
\hline 5 (Masa evacuable) & 3 & 2.2 & 9 & 16.6 & 10 & 28.5 \\
\hline 6 (Masa no evacuable) & 0 & 0 & 10 & 18.5 & 10 & 28.5 \\
\hline Total & 132 & 9.1 & 54 & 90 & 35 & 83.3 \\
\hline
\end{tabular}

En la tabla 8, se expone el destino de los pacientes tras la evaluación inicial. La gran mayoría de ellos fueron dados de alta o derivados a otros centros, internándose sólo el 5,6\%. El 0,5\% llegó con criterios de muerte encefálica. La mortalidad global fue del 1,56\%. Falleció un 13\% de los TCE moderados y un 36\% de los TCE graves (tabla 9).

\section{Discusión}

Hasta donde nuestra información es correcta, presentamos el primer estudio epidemiológico del TCE en Argentina, efectuado de forma prospectiva y sistematizada, con definiciones acotadas y con una población representativa del conjunto de la sociedad argentina actual. Creemos, por tanto, que nuestros datos son relevantes tanto como punto de referencia para otros estudios en el país o fuera de él, así como para modificar las condiciones actuales con medidas e intervenciones de prevención.

Efectuar un estudio basado en la población en forma prospectiva no es sencillo y representa una de las fortalezas de nuestro trabajo. Prueba de ello es que la gran mayoría de los estudios epidemiológicos publicados son de base hospitalaria. No obstante, nuestro trabajo podría tener limitaciones, que comentamos. Una primera crítica que se puede 
Tabla 7

Incidencia de hematomas intracraneales según gravedad, en números absolutos y porcentajes relativos. Los totales inferiores expresan el porcentaje de lesiones por categoría y los de la columna derecha el porcentaje para la totalidad del grupo

\begin{tabular}{|l|c|c|c|c|}
\hline Lesión & TCE Leve & TCE moderado & TCE grave & Total \\
\hline HSD & $2(0.001 \%)$ & $7(11.6 \%)$ & $7(14.2 \%)$ & $16(0.009 \%)$ \\
\hline HED & $1(0.001 \%)$ & $3(5 \%)$ & $1(2.3 \%)$ & $5(0.003 \%)$ \\
\hline HIC & 0 & $3(5 \%)$ & 0 & $3(0.001 \%)$ \\
\hline Total & $3(0.002 \%)$ & $13(21.6 \%)$ & $8(19 \%)$ & \\
\hline HSD: hematoma subdural, HED: hematoma extradural, HIC: hematoma intracerebral \\
\hline
\end{tabular}

Tabla 8

Destino tras evaluación inicial

\begin{tabular}{|l|c|c|}
\hline Destino & Número absoluto & Porcentaje \\
\hline Alta & 1080 & 70.1 \\
\hline Derivación & 303 & 19.7 \\
\hline Fuga & 56 & 3.6 \\
\hline Internación Sala & 51 & 3.3 \\
\hline Internación UTI & 36 & 2.3 \\
\hline Muerto al llegar & 8 & 0.5 \\
\hline
\end{tabular}

realizar a este estudio es haber calculado todas las variables sobre la base de la población del área de la ciudad asignada a nuestro hospital. En efecto, si bien ésta tiene las características socioeconómicas descritas, representativas del conjunto de la ciudad de Buenos Aires, la cifra de población difiere con respecto a la expuesta, ya que aumenta a cerca de 800.000 habitantes durante las horas de trabajo. Sin embargo, la incidencia horaria del TCE muestra que casi el $70 \%$ de los accidentes ocurren fuera de las horas de trabajo, por lo que el aumento de población en las horas pico tendría una repercusión mínima en los resultados.
Otra limitación de nuestro estudio sería la posibilidad de haber incurrido en subregistro de pacientes, ya que, como sugieren Torner y Shootman ${ }^{21,22}$, siempre existe la posibilidad de subregistro de pacientes, tanto de leves y graves, así como de fallecidos en la recepción hospitalaria. Dada la organización del sistema de salud de la Ciudad de Buenos Aires, todos los pacientes que presentan accidentes en la vía pública o consultan en su domicilio son derivados por el Sistema de Emergencias (SAME) al hospital de referencia, razón por la cual el subregistro es mínimo.

Finalmente, el tipo de clasificación elegida, en nuestro caso la GCS podría ser cuestionada. A pesar de sus limitaciones, hemos elegido la Escala de Glasgow por ser la más difundida, aceptada y con menor variabilidad entre observadores, ya sean hospitalarios o extrahospitalarios. La mayor parte de los trabajos epidemiológicos publicados de TCE adolecen, a nuestro juicio, de una clasificación adecuada, ya que han empleado la Clasificación Internacional de Enfermedades del Centro Nacional de Control de Enfermedades de Estados Unidos, pero en forma retrospectiva, lo cual, junto a ser muchos de ellos de base hospitalaria puede explicar las diferencias en los porcentajes de leves, moderados y graves.

Nuestros resultados muestran que la incidencia en nuestra área (322 por 100.000 habitantes) se sitúa dentro de valores previamente publicados, 90 a 400 casos anuales por

\section{Tabla 9}

Mortalidad

\begin{tabular}{|l|c|c|c|c|}
\hline & TCE total & TCE leve & TCE moderado & TCE grave \\
\hline Total & $23(1,56 \%)$ & 0,00 & $8(13,33 \%)$ & $15(35,71 \%)$ \\
\hline Hombres & $16(1,31)$ & 0,00 & $6(11,76 \%)$ & $10(31,25 \%)$ \\
\hline Mujeres & $7(2,48 \%)$ & 0,00 & $2(22,22 \%)$ & $5(50,00 \%)$ \\
\hline
\end{tabular}


Tabla 10

Incidencia, mortalidad y tasas de severidad en estudios basados en la población

\begin{tabular}{|c|c|c|c|c|c|c|c|c|}
\hline POBLACION & AÑo & MÉTODO & TIPO T & TASA $10^{5}$ & MORTALIDAD (\%) & LEVE (\%) & MODER.ADO (\%) & GRAVE (\%) \\
\hline Olmstead $^{2}$ & $65-74$ & Retrosp. & Pob. & 193 & 11 & 63 & 29 & 7 \\
\hline San Diego ${ }^{7}$ & 78 & Retrosp & .Pob. & 295 & 7 & 91 & 91 & 9 \\
\hline San Diego ${ }^{8}$ & 81 & Retrosp. & Pob. & 180 & 14 & 82 & 9 & 9 \\
\hline Charlottesville5 & 78 & Retrosp. & Hosp. & 208 & 7 & 49 & 26 & 25 \\
\hline Chicago (raza negra) ${ }^{9}$ & $79-80$ & Retrosp. & Hosp. & 403 & 8 & 86 & 9 & $5 \mathrm{I}$ \\
\hline Aquitaine $^{21}$ & 86 & Prosp. & Pob. & 134 & 8 & 80 & 11 & 9 \\
\hline Johanesburgo ${ }^{16}$ & $86-87$ & Prosp. & Hosp. & 91 & 22 & 88 & 7 & 5 \\
\hline Cantabria $^{24}$ & 88 & Retrosp. & Pob. & 316 & 25 & 88 & 8 & 5 \\
\hline Buenos Aires & 03 & Prosp. & Pob. & 322 & 1.34 & 93 & 4 & 3 \\
\hline
\end{tabular}

Modificado de Torner ${ }^{16}$. Prosp.: Prospectivo. Retrosp.: Retrospectivo. Pob.: Basado en población general. Hosp.: Basado en población hospitalaria

100.000 habitantes dependientes del área geográfica estudiada $^{6}$, aunque en sus tramos más altos. Así en los EEUU ${ }^{3,6}$ se sitúa, como en España, en 200 casos cada 100.000 habitantes por año. De ellos, en EEUU, aproximadamente 500.000 personas sufren un TCE grave, cifra que incluye los que requieren hospitalización (450.000) y aquellos que mueren antes de llegar al hospital (50.000). De los 450.000 que ingresan al hospital, 100.000 personas por año mantienen discapacidades residuales significativas. Estudios realizados en Inglaterra, Gales y Escocia mostraron tasas de 270 y 313 por $100.000^{6}$. Cifras parecidas se observaron en Francia, 281 por $100.000^{12,20}$ con un perfil diferente, comparando los dos trabajos, en el mecanismo de la lesión, con un aumento de las caídas y disminución de los accidentes de tránsito. En Italia, en 1998, mostraron tasas de 314 por $100.000^{17}$, similares al estudio de Johannesburgo (Sudáfrica) con $316 \mathrm{TCE}$ por $100.000^{15}$, mientras que en Samoa, Wallace ${ }^{24}$, halló en 1993 una incidencia menor (165 por 100.000 habitantes). Otros estudios recientes ${ }^{1,14,17,20,21}$, también retrospectivos, encuentran tasas de incidencia similares, con un perfil cambiante en cuanto al mecanismo del trauma.

Un dato particular de nuestro trabajo, al no haberse encontrado resultados similares en la literatura, es la diferencia de edad entre hombres y mujeres, significativamente mayor en las mujeres que podemos atribuir a la mayor longevidad de estas últimas y, por tanto, mayor posibilidad de exposición a traumatismos. La relación hombre / mujer, en cambio, se mantiene dentro de los parámetros descritos en los trabajos citados previamente ${ }^{3,4,10-15,16,17,19,20}$, coincidiendo las variaciones con la edad.

El resto de los hallazgos de nuestra investigación es coincidente con la literatura, tanto en los mecanismos del trauma como en la incidencia de hematomas intracraneales, si bien, nuestros resultados concuerdan más con los trabajos de la década del 70 que con los actuales, por la gran importancia en nuestra serie de los accidentes relacionados de tráfico. La agresión interpersonal representa la tercera causa de lesión, la cual, llamativamente, se mantiene en dicha posición en casi todas las publicaciones.

Respecto a la mortalidad, Torner y Shootman señalan que la mayor parte de las muertes ocurren previamente a la hospitalización de los pacientes. Estos autores observaron, en $1996^{22}$, una mortalidad de 1\% para TCE leve, $18 \%$ para moderado y $48 \%$ para TCE grave. En nuestra serie no hubo fallecidos en el grupo de leves y la mortalidad en los otros dos grupos de TCE fue menor a la de estos autores y en rangos análogos a otros ${ }^{6}$. Probablemente, la disparidad en la mortalidad se explique por la diferencia de diseño de nuestro estudio con la mayoría de los trabajos epidemiológicos sobre TCE, realizados en la década del 70 y 80 , retrospectivos, y con fallos en las definiciones. En la tabla 10 se resumen los principales hallazgos de esta investigación comparados con los de la literatura clásica.

Como conclusiones, este estudio señala que, en la comunidad argentina estudiada, la incidencia de TCE es elevada, con una proporción de TCE moderados y graves inferior a otras series. Los accidentes de tráfico continúan como primer agente causal, afectando, sobre todo, a la población joven masculina, mientras las caídas de altura y atropellos inciden en la población mayor de 40 años y sexo femenino. La tasa de mortalidad se encuentra dentro de los rangos actualmente admitidos. Finalmente, los datos aportados, pensamos, ofrecen una información valiosa para 
aunar esfuerzos médicos y políticos que mejoren la situación actual.

\section{Bibliografía}

1. Andersson, E.H., Bjorklund, R., Emanuelson, I, Stalhammar, D.: Epidemiology of traumatic brain injury: a population based study in western Sweden. Acta Neurol Scand. 2003; 107: 256-259.

2. Annegers,, J.F., Grabow, J.D., Kurland, L.t, Laws, E.D. Jr.: The incidence causes and secular trends of head trauma in Olmstead County, Minnesota, 1935-1974. Neurology 1980; 30: 912-919.

3. Finfer, S.R., Cohen, J.: Severe traumatic brain injury. Resuscitation 2001; 48: 77-90.30.

4. Gilli Miner, M., Murillo Cabezas, F., Perea Milla, E.: Epidemiología y prevención de los traumatismos. En: Martínez Navarro F, Antó JM, Castellanos PI., Gilli M, Marset P, Navarro V(eds.) Salud Pública. Madrid, McGraw-Hill Interamerica 1998.

5. Jagger, J., Levine, J., Jane, J. et al.: Epidemiologic features of head injury in a predominant rural population. J Trauma 1984; 24: 40-44.

6. Jennett, B., Mac Millan, R.: Epidemiology of head injury. Br Med J 1981; 282: 101-104

7. Klauber, M.R., Barret Connor, E., Marshall, L.F. et al.: The epidemiology of head injury. A prospective study of an entire community: San Diego, California, 1978. Am J Epidemiology 1981; 113: 500-509.

8. Klauber, M.R., Marshall, L.F., Barret Connor, E., Bowers, S.A.: Prospective study of patients hospitalized with head injury in San Diego County, 1978. Neurosurgery 1981;9: 236-241.

9. Krauss, J.F., Black, M.A., Hessol, N. et al.: The incidente of acute brain injury and serious impairement in a defined population. Am J Epidemiol 1984; 119: 186-201.

10. Lopez Bastida, J., Serrano Aguilar, P., Duque González, B.: The economic costs of traffic accidents in Spain. J Trauma 2004; 56: 883-889.

11. Management and Prognosis of Severe Traumatic Brain Injury. Part II: Early indicators of of prognosis in severe traumatic brain injury. Age. Brain Trauma Foundation (ed), 2000, pag 27-38.

12. Marshall, L; Marshall, S; Klauber, M et al.: A new classification of head injury based on computerized tomography. J.Neurosurg 1991:75(S): 14.

13. Masson, F., Thicoipe, M., Aye, P., et al.: Aquitaine Group for Severe Brain Injuries Study. Epidemiology of severe brain injuries: a prospective population-based study. J Trauma. 2001; 51: 481-489.

14. Meerhoff, S.R., de Kruijk, J.R., Rutten, J., Leffers, P., Twijnstra, A.: Incidente of traumatic head or brain injuries in catchment area of Academic Hospital Maastricht in 1997. Ned Tijdschr Geneeskd. 2000; 144:1915-1918. (Abstract).

15. Nell, V., Brown., D.S.O.: Epidemiology of traumatic brain injury in Johannesburg II. Morbidity, mortality and etiology. Soc Sci Med 1991; 33: 289-296.

16. Raja, I.A., Vohra, A.H., Ahmed, M.: Neurotrauma in Pakistan. World J Surg. 2001; 25:1230-1237.

17. Servadei, F., Verlicchi, A., Soldano, F., Zanotti, B., Piffer, S.: Descriptive Epidemiology of head injury in Romagna and Trentino. Comparison between two geographically different italian regions. Neuroepidemiology 2002; 21: 297-304,

18. The Study Group on Head Injury of the Italian Society for Neurosurgery. Guidelines for minor head injured patients' management in adult age. J Neurosurg Sci 1996; 40: 11-15.

19. Thornhill, S., Teasdale, G.M., Murray, G.D., McEwen, J., Roy, C.W., Penny, K.I.: Disability in young people and adults one year after head injury: prospective cohort study. BMJ. 2000; 320: 1631-1635.

20. Tiret, L., Haysherr, E., Thicoipe, M. et al.: Epidemiology of head trauma in Aquitaine (France), 1986: a community-based study of hospital admissions and deaths. Int J Epidemiol 1990; 19: 133-140.

21. Torner, J.C., Shootman, M.: Epidemiology of closed head injury en Textbook of head injury, Becker DP., Gudeman S. Philadelphia; W B Saunders Company. 1989

22. Torner, J.C., Shootman, M.: Epidemiology of Closed head Injury. In Rizzo M and Tranel D (eds). Head Injury and the postconcussive syndrome. Churchill Livingston, New York, 1996. Page 19-43.

23. Vázquez Barquero, A., Vázquez Barquero, J.L., Austin, O., et al.: The epidemiology of head trauma in Cantabria. Eur J Epidemiol 1992; 832-837.

24. Wallace, G.L.: Stroke and traumatic brain injury in Amerika Samoa. Hawaii Med J 1993; 52: 234-250.

Marchio, P.S.; Previgliano, I.J.; Goldini, C.E.; MurilloCabezas, F.: Traumatismo craneoencefálico en la ciudad de Buenos Aires: estudio epidemiológico prospectivo de base poblacional. Neurocirugía 2006; 17: 14-22.

Correspondencia postal: Ignacio J. Previgliano. Virrey Loreto 2676. $7^{\circ}$. CP 1426. Ciudad Autónoma de Buenos Aires. Argentina. 